\title{
LA NOCIÓN DE SECTOR PÚBLICO INSTITUCIONAL: APLAUSO, CRÍTICA Y DESCONCIERTO
}

\author{
Alejandra Boto Álvarez \\ Profesora Ayudante Doctora (acreditada PTU) \\ Universidad de Oviedo \\ botoalejandra@uniovi.es
}

\begin{abstract}
Aprovechando y agradeciendo la amable invitación cursada por los profesores Muñoz Machado y Rebollo Puig durante el desarrollo del III Seminario sobre la Reforma del Estado, me atrevo a plasmar aquí algunas reflexiones en torno a la novedad que supone, como categoría, la identificación de un "sector público institucional" en el artículo 2.2 del Proyecto de Ley de Régimen Jurídico del Sector Público, dentro del ámbito subjetivo de aplicación, que luego se concreta en los artículos 84 y siguientes para la dimensión estatal. Tales reflexiones pretenden contribuir a un debate conceptual, aunque con importantes implicaciones prácticas, que no ha dejado de interesarme desde las investigaciones doctorales que acabaron plasmándose en la monografía La Administración instrumental en el proceso (2011, Madrid: Reus). Como se verá, la tesis allí contenida sobre la determinación formal y material de las personificaciones instrumentales es el parámetro con el que acometo el balance siguiente. Intentaré hacerlo de manera telegráfica y ágil, de ahí la ausencia de notas al pie y referencias bibliográficas; como se hubiese esperado de una comunicación oral a la que me sentí cohibida in situ ante la consciencia de no estar ni remotamente a la altura de las brillantes observaciones de los ponentes, ni de las sugerentes intervenciones posteriores.
\end{abstract}

\section{EL ESFUERZO DELIMITADOR: APLAUSO}

Desde hace ya tiempo, la fórmula "sector público" resulta cada vez más utilizada en la legislación positiva, seguramente por entenderla de utilidad creciente dado el progresivo difuminado de la frontera que separa lo público y lo privado, y la multiplicación de supuestos en que normas del Derecho Administrativo se aplican a personificaciones jurídico-privadas de la Administración. En esta ocasión, el término se hace presente desde el mismo título de la reforma normativa y, sin embargo, la versión del Anteproyecto que se sometió a información pública empleaba la expresión desde su primer artículo sin ninguna presentación previa o esfuerzo de delimitación, como si se tratase de una única realidad jurídica transparente, conocida y consensuada. La difícil transición de la noción de sector público a la de Administración pública que se ponía de manifiesto en aquel texto, y que no pasó desapercibida en el informe que sobre el mismo emitió en el mes de marzo el CGPJ, fue final y felizmente atajada antes de someter poco después el Anteproyecto al dictamen del Consejo de Estado, tal y como puede comprobarse cotejando las versiones del artículo 2 del Anteproyecto que ambos órganos comentan.

En el artículo 2.1 del Proyecto de Ley, el sector público se define como integrado por la Administración General del Estado, las Administraciones de las Comunidades Autónomas, las Entidades que integran la Administración Local y el sector público institucional, noción que a su vez se desgrana en el apartado siguiente y sobre cuyo estudio tornaremos a continuación. El sector público así definido es el ámbito subjetivo de aplicación de la Ley, pero estamos por primera vez ante un concepto de vocación general, cuya definición no aparece ceñida, como es habitual en otras leyes que también incorporan al sector público en su título, a los solos efectos de la norma en cuestión.

En este sentido, la concreción de una definición del sector público primero y del sector público institucional después que acomete el artículo 2 no puede merecer sino mi felicitación. Y es que, como ya he sostenido en otras ocasiones, los esfuerzos de racionalización organizativa deben acometerse en sede de la legislación organizativa, y no en ámbitos sectoriales que pueden no tener los mismos perfiles jurídicos, de acuerdo con lo que apuntara García-Trevijano Fos ya en 1970. 


\section{LA IDENTIFICACIÓN CONCRETA: CRÍTICA}

La noción de sector público institucional es así un componente del sector público en general y se integra, conforme al artículo 2.2 del Proyecto de Ley, por:

\section{a) Los organismos públicos y entidades de derecho público vinculados o dependientes de las Administraciones públicas}

El concepto de Administración pública se define en el artículo 2.4 de forma aparentemente tautológica, pues incluye, además de a los entes del sector público que no son sector público institucional, a este mismo primer subtipo de entes institucionales. No es, en definitiva, sino la prueba de que en lo gráfico no nos movemos aquí con tres categorías subjetivas concéntricas, al estilo de la normativa contractual, sino de un círculo amplio (el sector público) que engloba dos secantes (Administración pública y sector público institucional). Que las personificaciones instrumentales de derecho público tienen que quedar incluidas en esa zona de intersección parece fuera de toda duda y supone muy poco de innovador si se coteja con el artículo 2 de la Ley 30/1992. Podría, por eso mismo, seguir subsistiendo la vieja polémica sobre la ubicación de entidades con personalidad jurídico-pública pero régimen jurídico parcialmente privado. A mi juicio y de acuerdo con una abrumadora jurisprudencia, el debate es estéril y no puede haber dudas de que los identificados como organismos bifrontes son sustantivamente Administraciones por su personalidad jurídico-pública, aunque puedan no serlo a efectos sectoriales concretos por otra delimitación legislativa específica.

En todo caso, debe lamentarse que el legislador haya dejado pasar, una vez más, la ocasión de concretar que una "entidad de derecho público" no es otra cosa sino una entidad con personalidad jurídica de derecho público y no siempre una entidad con régimen jurídico de derecho público. Esta definición, por último, sería además ejercicio de una mejor técnica legislativa, pues la remisión a lo que sea un "organismo público" sin mayores precisiones resulta ambigua, máxime cuando no existe ningún precepto que unifique lo que tal categoría, de larga tradición en el ámbito estatal, representa con carácter básico. Si la expresión "organismo público" desapareciese de este primer inciso de la enumeración, no sólo no se perdería información (sin saber lo que sea exactamente en todos los ámbitos sí parece claro que es una especie dentro del género de las entidades de derecho público), sino que además abonaría un total paralelismo con la forma en que queda redactado el segundo componente del sector público institucional en el mismo artículo y que también merece comentario.

\section{b) Las entidades de derecho privado vinculadas o dependientes de las Administraciones públicas}

Tal y como ha puesto de manifiesto el Consejo de Estado, este apartado parece referido a las sociedades mercantiles y fundaciones del sector público. En principio debo aplaudir su consideración dentro del ámbito del sector institucional por considerarlas como entidades contingentes, creadas buscando la gestión más eficaz por la flexibilidad de régimen jurídico que comportan, con fines sectorialmente especializados y autonomía funcional para lograrlos, no vinculadas por una relación de jerarquía pero controladas por la Administración que las crea y, desde luego, con un campo de acción y unos intereses propios. Creo firmemente que participan de la misma esencia de la tradicional Administración instrumental salvo del carácter jurídico-público; no son Administración, pero pertenecen al bloque instrumental de lo público.

Dicho esto, debo sin embargo criticar la configuración del Proyecto de Ley en dos aspectos. El primero, comentado ya en su ponencia por el profesor Sánchez Morón y por tanto en el que no me detendré en exceso, tiene que ver con la ausencia de una diferenciación entre las sociedades de capital íntegramente público y las que cuentan con socios privados. No solamente en estas últimas la presencia del interés privado puede matizar el actuar de la entidad debilitando la relación de instrumentalidad, o en las primeras suscitarse problemas de relaciones interadministrativas cuando concurran varios socios públicos, sino que la eventual atribución a unas y otras del ejercicio de "potestades administrativas" sin más cautelas convierte la redacción en un dislate. Además, en relación con la base teórica, echo en falta una referencia en este punto, de nuevo, a la personalidad jurídica, que en este caso por responder a una de las formas tradicionalmente civilistas o mercantilistas es lo que determina el adjetivo de privado al derecho de pertenencia de la entidad. También me confieso nostálgica de la idea de la descentralización funcional como principio que justifica la imbricación de sociedades y fundaciones en la organización pública. Cierto es que, como apuntó en su ponencia el profesor Jiménez-Blanco, la referencia a la "nueva" descentralización de Royo Villanueva puede parecer trasnochada, pero no menos cierto es que resulta susceptible de leerse en clave actualizada, como encomiablemente ha hecho también con otros juristas de la época el profesor Sosa Wagner. Además, más allá de las parole, es un origen conceptual que si no se pierde de vista puede facilitar considerablemente un análisis de derecho comparado, por ejemplo. 
Literalmente, el Proyecto no impone la descentralización funcional como principio general de actuación del sector público pues no aparece como tal en el artículo 3, que se refiere además a los principios de actuación de las "Administraciones públicas" y no distingue entre los tipos de descentralización. Tan sólo aparece a partir del artículo 54, como un principio propio del funcionamiento de la Administración General del Estado entremezclado además en ocasiones con inquietantes equiparaciones a la "independencia" (artículo 88) sobre las que volveremos más adelante.

\section{c) Las Universidades públicas}

Este inciso ha sido profundamente criticado por el Consejo de Estado por entender, en primer lugar, que no tiene sentido incluir a las Universidades públicas, que no están sometidas a órdenes e instrucciones de la Administración territorial de referencia, junto a entidades que están sujetas a una relación de dependencia o de instrumentalidad; y en segundo lugar, que tal inclusión resulta forzada porque la regulación proyectada para el sector público institucional sólo es supletoria respecto de ellas. El propio Consejo de Estado asume que la única razón que puede justificar esta inclusión es la sujeción de las Universidades públicas a los controles de eficacia y supervisión continua que establece la nueva regulación.

No podría estar más de acuerdo, añadiendo a las razones esgrimidas por el supremo órgano consultivo una adicional de corte teórico que engarza con la evocación antes realizada de la descentralización funcional como base conceptual de los entes institucionales. En efecto, como ya señalara el profesor Fernández Rodríguez, la autonomía universitaria no es una autonomía instrumental porque esta responde a necesidades técnicas (de gestión y de eficacia), mientras que la autonomía universitaria presenta de raíz un espíritu distinto: la autonomía y libertad para la Ciencia, para la enseñanza y la investigación. De forma más prosaica puede decirse que las Universidades no pueden ser consideradas entes instrumentales porque no presentan las notas características de esta categoría; así por ejemplo, no existe ningún tipo de relación de adscripción con órganos estatales ni autonómicos y tienen marcadas potestades de autogobierno. El hecho de que su autonomía no responda a razones coyunturales, ni su existencia sea contingente puesto que está sustentada en el artículo 27.10 de la Constitución, no son sino argumentos a mayor abundamiento. Las Universidades no están, pues, sujetas a una relación de instrumentalidad. Sin embargo, es cierto que tanto el Estado como fundamentalmente las Comunidades Autónomas tienen amplios poderes de injerencia sobre ellas que se detallan a lo largo del articulado de su Ley Orgánica; por eso puede en ocasiones hablarse de ellas como "entes vinculados" a las Administraciones territoriales. Pero no estamos ante Administraciones instrumentales, sino ante una realidad cualitativamente distinta, que tiene un fundamento que no es la descentralización funcional y la eficiencia, sino la autonomía universitaria constitucionalmente reconocida. Lo que ocurre, en definitiva, es que las Universidades sencillamente no son encasillables dentro de los tipos organizativos clásicos.

De lo que en cambio no puede dudarse es de su carácter de Administraciones públicas, que sin embargo en la redacción del Proyecto de Ley se niega. En este punto, criticado ya en su ponencia por el profesor Santamaría Pastor, sólo cabe sorprenderse ante la mutación de naturaleza que se ha producido respecto de la versión del Anteproyecto sometida a información pública, que claramente disponía la consideración de Administración de las Universidades, regidas por su normativa específica y supletoriamente por las previsiones del nuevo texto legal. Qué pueda haber ocurrido para motivar el cambio de criterio, y cómo este ha arrastrado también a la definición contenida en el Proyecto de Ley del procedimiento administrativo, es algo sobre lo que quizás convendría indagar.

Hasta aquíla enumeración de tipologías que teóricamente agota la definición genérica del artículo 2.2 del Proyecto de Ley. Digo teóricamente porque como resulta evidente, el Proyecto sigue sin poder solucionar el eterno problema de la insuficiencia de rango de las normas de organización y la ampliación del inicial numerus clausus por leyes posteriores, e incluso por las propias disposiciones adicionales del mismo texto.

Finalmente llama la atención, además de lo ya comentado, lo que está ausente. Así, como se verá a continuación, hay entes que se identifican dentro del sector público institucional estatal que sin embargo no tienen referencia en el general. Además, el resultado final no parece acorde con los grandilocuentes anuncios llevados a cabo en su día por el informe CORA, cuando propugnaba que se acometería la determinación de un marco normativo común, mínimo pero básico, para ordenar la dispersión y heterogeneidad del sector institucional y que abordaría, entre otras cosas, los criterios identificativos de cada tipo de ente institucional. El paso atrás seguramente tiene que ver con legítimas dudas sobre la constitucionalidad de tal medida teniendo en cuenta la doctrina del Tribunal Constitucional sobre el reparto de competencias organizativas entre el Estado y las Comunidades Autónomas. No me prodigo ahora en este aspecto, merecedor por si sólo de reflexión autónoma. 


\section{El sector público institucional estatal: perplejidad}

La composición y clasificación del sector público institucional estatal se contiene en el artículo 84 del Proyecto de Ley. Hasta cierto punto es lógico y legítimo que sea una regulación más puntillosa y detallada que la del sector institucional general, pero como ya se ha anticipado, lo que resulta cuanto menos chocante es que aparezcan categorías totalmente nuevas, no contempladas en el artículo 2.2.

Según tal artículo 84, el sector público institucional estatal está integrado por:

\section{a) Los organismos públicos, que se clasifican en Organismos Autónomos y Entidades Públicas Empresariales}

No me detendré en exceso en este punto, ya comentado por los ponentes y desgranado en profundidad en el dictamen del Consejo de Estado. Como es evidente, tiene poco de novedoso más allá de suponer un retorno a la dicción original de la LOFAGE y acometer una supresión de la categoría de las Agencias Estatales que poco extraña a quien haya seguido con mínima atención el devenir de lo que ilusoriamente estaba llamado en 2006 a convertirse en una categoría general y única. Pero sin ser del todo sorprendente esta aféresis, tampoco sería justo convertirla en crónica anunciada. En efecto, y hasta donde he podido entender, el informe CORA era poco proclive a la institución, pero no llegaba a propugnar directamente su eliminación e incluso en la doctrina hay quien había sostenido la conveniencia de ensayar una fórmula similar en los ámbitos autonómico y local.

Harto curioso resulta el inciso final del artículo 88 cuando al referirse a las razones organizativas de los organismos públicos estatales se hace referencia a la descentralización funcional (ipor fin!), pero también, alternativamente, a una "independencia" cuyo alcance no se explica. La expresión sólo se entiende, y así todo sólo en parte por lo que luego se comentará, como reminiscencia in transito que indebidamente ha sobrevivido al cambio de criterio respecto a las conocidas como Administraciones independientes del Anteproyecto al Proyecto de Ley.

\section{b) Las autoridades administrativas independientes}

La primera aparición de este tipo de entidades en el Proyecto de Ley da pie al planteamiento de numerosas cuestiones que no han pasado desapercibidas para los ponentes del Seminario ni tampoco para el Consejo de Estado. Con casi todas ellas estoy, como no, de acuerdo, en particular en lo relativo a la observación del profesor Sánchez Morón sobre la imperfecta definición del ámbito de actuación de estas autoridades. Realizaré además alguna observación adicional.

Para comenzar, me surgen los primeros interrogantes con la terminología empleada, que evita la doctrinalmente clásica denominación de "Administraciones independientes" decantándose por la de "autoridades administrativas independientes"; puede parecer una mera preferencia estilística, pero desde el momento en que las mismas no tienen encaje específico en la definición de Administración del artículo 2.4 del mismo Proyecto, el debate sobre su verdadera naturaleza está servido. No ocurría esto en el Anteproyecto, ni en la versión que se sometió a información pública ni en la que estudió el Consejo de Estado a juzgar por las referencias que contiene su Dictamen. Aquel texto contemplaba estas entidades como un subtipo de organismos públicos junto a los Organismos Autónomos y las Entidades Públicas Empresariales. Esta inclusión conceptual pareció una aberratio al Consejo de Estado, pero al menos dejaba clara la condición de Administración pública de estas autoridades. Además, no sería la primera vez que normativamente se configura una Administración independiente como un organismo público; así lo hace, si bien reconociendo una cierta atipicidad, el artículo 1 de la Ley 3/2013 respecto a la Comisión Nacional de los Mercados y la Competencia.

Particularmente entiendo que está justificado un tratamiento diferenciado para estas autoridades, al margen del propio de los organismos públicos clásicos, pero sin negar que estemos ante verdaderas entidades instrumentales. Este punto, que no queda siempre claro en el Dictamen del Consejo de Estado sobre el Anteproyecto, creo que puede sostenerse sin tapujos: las autoridades independientes sí deben formar parte del sector público institucional. Y es que, frente a lo sostenido más arriba respecto de las Universidades públicas, se trata de entidades que se crean por descentralización funcional, de existencia contingente, y que se caracterizan porque la vinculación con el ente de adscripción, que todas tienen, está atenuada pero sigue existiendo a pesar de su denominación de “independientes". El propio Consejo de Estado, en el expediente 1805/1997, ya reconoció que la posición institucional del Consejo de Seguridad Nuclear no era la misma que la de la Universidad Carlos III, por ejemplo. Ambas tienen en común, empero, el ser entidades no "dependientes" pero sí "vinculadas" a las Administraciones territoriales.

Que las autoridades independientes sean o no parte de la categoría de los organismos públicos es opinable, y aceptable a mi juicio siempre que se reconozcan, como ya apuntara el profesor López Menudo en un seminario 
de análisis contemporáneo a la LOFAGE, como unos organismos públicos específicos que estarían situados en un nivel más bajo del común dentro de la escala de instrumentalidad.

Por último, el hecho de que estas autoridades no aparezcan mencionadas hasta el artículo 84 lleva a interrogarse sobre su eventual existencia como parte de un sector público institucional no estatal; algo que el legislador no parece contemplar, dado su mutismo al respecto en el artículo 2.2. No extrañan por ello las acérrimas críticas del voto particular al informe del CGPJ sobre el Anteproyecto, que lo acusaban de acometer una velada reforma de la organización política del Estado, vertidas sin embargo con referencia a otros pasajes del texto.

\section{c) Las sociedades mercantiles estatales, y e) Las fundaciones del sector público}

Muy poco de novedoso hay en la regulación relativa a estos entes. Además de los extremos comentados durante el Seminario por la profesora de los Mozos Touya, llama la atención la ausencia, en sede de sociedades, de la fijación de reglas básicas relativas a la adscripción que sin embargo sí se determinan respecto de las fundaciones. Parece como si las primeras fuesen de composición siempre mucho más estable e indiscutida que las segundas.

\section{d) Los consorcios}

Desconcierto es la palabra para definir, una vez más, el encuentro tan tardío con esta figura en el texto del Proyecto de Ley, puesto que en apariencia de nuevo estas entidades se contemplan tan sólo como partes integrantes del sector público institucional estatal y no del general. Digo en apariencia porque el texto contiene profusas reglas para determinar la adscripción de los consorcios, resultando por ello que podrán existir también en otros niveles de sector público y es de suponer que en tal caso su naturaleza institucional no habría de mutar.

En efecto, parece claro que los consorcios nacen con la voluntad de ser prolongaciones funcionales de las Administraciones que en ellos se consorcian, dotados de personalidad jurídica propia para ejecutar mejor sus políticas. La razón de la existencia de estos entes recuerda pues a la que está en la base de la descentralización funcional: alcanzar una dimensión organizativa más adecuada a las necesidades económico-sociológicas, y que permita, en consecuencia, el logro de mejores resultados de forma más eficiente. En esta sede, tal dimensión organizativa se consigue uniendo las fuerzas de las Administraciones consorciadas. Será necesario precisar, eso sí, que en este caso la instrumentalidad debe entenderse también de forma rebajada: como una vinculación de conexión o enlace entre el consorcio y las Administraciones que lo integran, más que como una subordinación o dependencia al uso. La preocupación por el control presupuestario y de eficacia ha dado lugar a que de forma sucesiva el legislador se haya preocupado por la determinación de una relación de adscripción, incluso.

Tal adscripción lleva aparejada la aplicación de unas u otras reglas de presupuestación, contabilidad, control y personal, siendo susceptible de condicionar, a su vez, el propio sector institucional del consorcio. Así, por ejemplo, de la adscripción de un consorcio a una entidad local se deriva, por la sujeción a normas imperativas para todas estas (como el ejercicio de funciones reservadas a los funcionarios con habilitación de carácter estatal), la imposibilidad que aquel se dote de medios propios instrumentales en aplicación de lo previsto en el apartado tres de la nueva redacción de la disposición adicional novena de la Ley 7/1985 tras la reforma de 2013. Es comprensible por tanto que, tal y como dispone el Proyecto de Ley, un cambio de adscripción conlleve una revisión de los estatutos del consorcio.

\section{f) Los fondos sin personalidad jurídica}

El desconcierto previo troca ya en verdadera estupefacción al aparecer aquí, exclusivamente para el ámbito estatal, un integrante del sector público institucional no ya sólo que no estaba contemplado con carácter general en el artículo 2 del Proyecto de Ley, sino que incluso carece de subjetividad propia. Como bien ha señalado el Consejo de Estado, tales fondos ni siquiera son una unidad organizativa orgánica, sino tan sólo masas patrimoniales. La inclusión de los mismos en el sector institucional estatal sólo tiene sentido porque se quieran sujetar al régimen de control presupuestario, pero el resto de medidas previstas para el sector, pensadas para entes dotados de personalidad jurídica propia, difícilmente resultarán operativas.

Estos fondos han sido identificados como la forma más moderna de "huida del presupuesto" y en la actualidad su régimen económico-financiero, contable y de control se contiene en la Ley General Presupuestaria y en la Ley del Patrimonio de las Administraciones Públicas. Entiendo que en tal sede deberían quedar, visto no ya sólo que no responden al principio de descentralización funcional, sino que ni siquiera son técnicas organizativas.

La idea de experimentar con fórmulas organizativas sin personalidad jurídica no me resulta ajena; pero me espeluznaría incluirlas sin más cautelas como entidades del sector institucional, aunque sólo sea porque no creo ser la única docente que padece dificultades para hacer interiorizar en las aulas la diferencia entre un órgano y un organismo, y no quisiera contribuir más a la desazón de nuestros alumnos. 
En efecto, en ocasiones he defendido la utilidad de poner en marcha unidades organizativas institucionales sin personalidad, figura inspirada en el ordenamiento norteamericano. Con tal híbrido entre la descentralización funcional y la desconcentración (unidades con cierta autonomía y flexibilidad y un funcionamiento según las modernas reglas de la transparencia y la evaluación por resultados, pero sin personalidad jurídica propia ni una autonomía inamoviblemente configurada) podrían colmarse creo muchas de las aspiraciones de diversificación e identificación distinta de determinados entes, sin quebrar la unidad de régimen jurídico y de imputabilidad administrativa. Se reservaría así la personalidad jurídica diferenciada para aquellos entes descentralizados que verdaderamente precisan de ella por su funcionamiento autónomo más allá de la mera instrumentalidad, y se allanaría la simplificación de organigramas y la transparencia en la gestión.

No me atrevo a afirmar que tales unidades sin personalidad debieran incluirse en el inventario de entes del sector público, como ahora ocurre con los fondos sin personalidad. Creo que todo depende de la utilidad y unidad conceptual que quiera darse a tal inventario, que quizás entonces debiera trocar su nombre. Esas unidades sin personalidad requerirían, en todo caso, reglas distintas y una subcategoría propia.

Tampoco estoy propugnando la vuelta a la creación de "servicios administrativos sin personalidad" -curioso bucle en el tiempo estaría dándose si en pleno siglo XXI no sólo reviviese como se ha dicho el modelo de la Ley de Régimen Jurídico de la Administración del Estado de 1957, sino también el de la Ley de Entidades Estatales Autónomas de 1958. Las unidades a las que me refiero presentan similitudes con los viejos servicios sin personalidad sí, pero al mismo tiempo son algo más que esto y sobre todo, frente a los fondos cuya consideración como sector institucional estoy criticando, son verdaderas técnicas de organización.

En lo sustantivo, para acabar, la única novedad que aporta el Proyecto de Ley en la regulación de los fondos carentes de personalidad jurídica del sector público estatal es la obligación de que en su denominación aparezca de forma expresa la indicación de su naturaleza. Es una previsión idéntica a la que en el mismo Proyecto aparece respecto a los medios propios, sin que como es lógico éstos se hayan configurado como una categoría propia dentro del sector público institucional, entre otras razones porque también pueden estar fuera de él, como sostengo que debería pasar con los fondos sin personalidad.

\section{g) Las Universidades públicas no transferidas}

La consideración de la UIMP y la UNED como entes del sector público institucional distintos de los organismos públicos y sin la consideración de Administración pública, aunque criticable por muchas razones ya apuntadas, resulta al menos conforme con la lógica del Proyecto de Ley desde su artículo 2. Sin entrar en el debate sobre la segunda de las exclusiones, la primera es del agrado del Consejo de Estado, por entender el reconocimiento de su posición institucional al margen de los organismos públicos "conforme a su autonomía constitucional reconocida".

Es preciso matizar sin embargo que existe para el caso concreto de UIMP y UNED una previsión positiva que opera como límite en tanto no sea derogada y que siempre he entendido como una excepción a la naturaleza de las Universidades que, como sostengo más arriba, pienso que no pueda reconocerse instrumental en general. El Proyecto de Ley, sin embargo, parece obviar esta barrera.

A la UNED se refiere la disposición adicional segunda de la Ley Orgánica de Universidades, que le reconoce una regulación específica; sus actuales estatutos (Real Decreto 1239/2011) la definen en el artículo 1 como "una institución de derecho público, de las contempladas en la disposición adicional décima de la LOFAGE"; casi huelga recordar que tal disposición en la LOFAGE lleva por título el de "Régimen jurídico de determinados organismos públicos". Cierto es que la derogación normativa que contempla el Anteproyecto podría eliminar el obstáculo, pero también lo es que la disposición transitoria segunda contempla un principio de continuidad enmarcado en el plazo de la adicional cuarta. En todo caso, con mayor rotundidad y rango normativo, a la UIMP se refiere la disposición adicional tercera de la Ley Orgánica de Universidades, estableciendo en el párrafo segundo de manera literal que mantiene su carácter de organismo autónomo.

Termino ya mis reflexiones que, aunque han ocupado seguramente más de lo deseable, se centran únicamente en los aspectos formales de determinación de las categorías de sector público institucional y sector público institucional estatal. Sobre el régimen material mucho queda por decir, pero habrá de posponerse para mejor ocasión, si es que finalmente el Proyecto de Ley llega a materializarse. 